\title{
1 Multiple causal DNA variants in a single gene affect gene 2 expression in trans
}

3 Sheila Lutz, Krisna Van Dyke, Frank W. Albert

4 Department of Genetics, Cell Biology, \& Development

5 University of Minnesota

6 Minneapolis, MN 55455

7 USA

8 Correspondence to: falbert@umn.edu

\section{Abstract}

10 Identifying the specific causal DNA differences in a genome that contribute to variation in

11 phenotypic traits is a key goal of genetic research. Trans-acting DNA variants that alter gene

12 expression are important sources of genetic variation. Several genes are known to carry single

13 causal variants that affect the expression of numerous genes in trans. Whether these single

14 variants are representative of the architecture of trans-acting variation is unknown. Here, we

15 studied the gene $I R A 2$, which carries variants with broad, trans-acting effects on gene expression

16 in two strains of the yeast Saccharomyces cerevisiae. We found that IRA2 contains at least seven

17 causal nonsynonymous variants. The causal variants were located throughout the gene body and

18 included a pair of neighboring variants with opposing effects that largely canceled each other

19 out. The causal variants showed evidence for non-additive epistatic interactions, in particular

20 among variants at the 5' end of the gene. These results show that the molecular basis of

21 trans-acting variation can involve considerable complexity even within a single gene. 


\section{Introduction}

23 Genetic differences among individuals contribute to variation in phenotypic traits. The

24 identification of causal DNA variants that influence a given trait among the vast majority of

25 variants thought to have no effect is a central challenge in genetics (Rockman, 2012).

26 Quantitative trait locus (QTL) mapping has been used in thousands of studies to identify

27 genomic regions that contain causal variants (Mackay et al., 2009). However, the resulting QTLs

28 are usually broad and span multiple genes and numerous variants. Efforts to identify the molecular cause of a QTL often implicitly assume that the QTL contains only a single causal variant that can be "fine-mapped" through a series of experiments, aided by annotations of gene function and, more recently, computational predictions of variant effect (Adzhubei et al., 2013; Choi and Chan, 2015; Kircher et al., 2014; Sim et al., 2012). Well-known success stories demonstrate that single causal genes and variants can be identified in QTLs (Carneiro et al.,

35 At the same time, the assumption that a typical QTL harbors a single causal variant has frequently turned out to be incorrect (Flint and Mackay, 2009). For example, a single QTL influencing the ability of yeast to grow in high temperature was shown to harbor three different causal genes (Steinmetz et al., 2002). A single chromosome region in C. elegans contained multiple independent effects on growth and reproduction (Bernstein et al., 2019). Approximately $40 \%$ of QTLs for various physiological traits identified in an outbred rat population were estimated to be due to multiple causal variants (Rat Genome Sequencing and Mapping Consortium, 2013). Some of the most finely dissected QTLs affect traits in the yeast Saccharomyces cerevisiae (Fay, 2013). In several yeast QTLs, single genes were found to carry multiple causal variants (Fidalgo et al., 2006; Gerke et al., 2009). Whether typical QTLs tend to be caused by one or multiple variants in one or multiple genes remains an open question, especially given experimental biases that benefit identification of single variants with strong effect compared to multiple variants with smaller effects (Rockman, 2012).

48 Gene expression variation is a key bridge between DNA variation and organismal phenotypes (Albert and Kruglyak, 2015). In particular, work in yeast crosses (Albert et al., 2018; Brem et al., 2002) and human populations (Grundberg et al., 2012; Wright et al., 2014) revealed important contributions of variants with trans-acting effects on gene expression. Trans-acting DNA variants change the activity or abundance of a regulatory factor. This factor influences the expression of other genes, which can be located anywhere in the genome. In yeast crosses, trans-acting variation arises almost exclusively from "hotspot" regions (Albert et al., 2018) that contain DNA variants that alter the abundance of many genes. Trans-acting hotspots are commonly identified by QTL mapping of mRNA abundance as the phenotype of interest. 58 and contain numerous DNA variants. 
59 Due to their broad effects on expression, trans-eQTL hotspots have served as models for

60 understanding trans-acting regulatory variation. Several yeast hotspots have been dissected to the

61 causal gene (Brion et al., 2013; Lewis and Gasch, 2012; Smith and Kruglyak, 2008) and to single

62 causal variants per gene (Brem et al., 2002; Brown et al., 2008; Fehrmann et al., 2013; Kim et

63 al., 2009; Lutz et al., 2019; Sudarsanam and Cohen, 2014; Yvert et al., 2003; Zhu et al., 2008).

64 Whether these results are representative of most trans-eQTL hotspots is unknown.

65 One particularly prominent trans-eQTL hotspot is caused by variation in the IRA2 gene (Smith

66 and Kruglyak, 2008). IRA2 encodes a regulator of RAS signaling. Together with IRA1, it is one

67 of two paralogous genes that encode the yeast homologs of the human Neurofibromin (NF1)

68 gene, mutations in which can cause neurofibromatosis 1, a disease characterized by uncontrolled

69 cell growth (Ballester et al., 1990; Ratner and Miller, 2015). In a cross between the laboratory

70 strain "BY" and the vineyard isolate "RM", IRA2 resides in a trans-eQTL hotspot that affects up

71 to 1,240 genes (Albert et al., 2018). Previous work showed that IRA2 is a causal gene in this

72 hotspot (Smith and Kruglyak, 2008). In addition to mRNA abundance, the IRA2 locus also

73 affects protein levels for many genes (Albert et al., 2014; Großbach et al., 2019), as well as yeast

74 growth in several different environmental conditions (Bloom et al., 2013; Breunig et al., 2014;

75 Wang and Kruglyak, 2014). Variation in IRA2 may also underlie QTLs for a variety of traits in

76 strains other than BY and RM (Stojiljkovic et al., 2020; Wang et al., 2019).

77 In spite of these broad effects on many traits, the causal variant or variants in IRA2 remain

78 unknown. In part, this is due to the large size of IRA2. With a length of 9.24 thousand bases $(\mathrm{kb})$,

79 the protein-coding IRA2 open reading frame (ORF) is one of the ten longest ORFs in the yeast

80 genome (Cherry et al., 2012), complicating allelic engineering.

81 Here, we used CRISPR-Swap, an efficient method for yeast genome engineering we recently

82 developed (Lutz et al., 2019), to dissect the molecular basis of the IRA2 hotspot in the BY / RM

83 yeast cross. We show that the IRA2 protein coding sequence contains at least seven causal

84 nonsynonymous variants and present evidence that these variants interact with each other in a

85 non-additive, epistatic manner. These results are in contrast to the simpler molecular basis of

86 other trans-eQTL hotspots dissected so far, highlighting that even a single causal gene can

87 harbor a complex genetic architecture. 


\section{Results}

\section{Protein-coding variation in $I R A 2$ affects gene expression in trans}

90 The profound trans effects of the IRA2 locus on the mRNA and protein levels of many genes

91 motivated us to identify the nucleotide variant(s) causing these effects. Variants in the coding

92 region of IRA2 were previously shown to affect the expression of numerous genes (Smith and

93 Kruglyak, 2008). However, the specific coding variants that are responsible for these effects are

94 unknown.

95 Comparison of the IRA2 alleles of BY and RM identified 26 nonsynonymous and 61

96 synonymous variants spread throughout the coding region (Figure 1A). Comparison with the $S$.

97 cerevisiae sister species $S$. paradoxus and an $S$. cerevisiae isolate from Taiwan that is presumed

98 to be an evolutionary outgroup to most other strains in this species (Peter et al., 2018) indicates

99 that 19 of the nonsynonymous variants are derived in RM, while 7 are derived in BY (Table 1).

100 Causal variants may be expected to occur at sites that are conserved across evolution and encode

101 amino acids with different chemical properties. To ask if any of the 26 nonsynonymous variants

102 are candidates for causing the IRA2 effect based on these criteria, we analyzed them using the

103 Protein Variation Effect Analyzer (PROVEAN) tool (Choi and Chan, 2015). Four of the derived

104 nonsynonymous variants were marked as "deleterious" (Table 1). Variants with strong effects on

105 phenotypes tend to be at low population frequency (Bloom et al., 2019; Fournier et al., 2019).

106 However, only two of the nonsynonymous variants are relatively rare in the population, with a

107 derived allele frequency of $10 \%$ or less, and neither of these were predicted to be deleterious

108 (Table 1). Together, this information did not pinpoint an individual variant as the obvious source

109 of the effects arising from IRA2.

110 The Ira2 protein negatively regulates the cAMP-PKA signalling pathway by stimulating

111 conversion of RAS GTPase (Ras1,2) from the active GTP-bound form to the inactive

112 GDP-bound form (Figure 1B). RAS-GTP activates adenylate cyclase (Cyr1) to produce cAMP.

113 High cAMP results in activation of Protein Kinase A (PKA) and subsequent phosphorylation of

114 its targets (Thevelein and de Winde, 1999). For example, high levels of cAMP reduce the stress

115 response by increasing the phosphorylation and cytoplasmic retention of Msn2,4, which in turn

116 leads to decreased expression of genes containing stress-response elements (STRE) (Görner et

117 al., 1998; Martínez-Pastor et al., 1996). One such gene, GPH1, encodes glycogen phosphorylase,

118 an enzyme required for the breakdown of glycogen (Wohler Sunnarborg et al., 2001). The

119 expression of GPH1 mRNA and Gph1 protein is strongly affected by the IRA2 locus, with the

$120 \mathrm{RM}$ allele resulting in higher expression (eQTL LOD $=21$, protein QTL LOD $=60$ ) (Albert et

121 al., 2018, 2014). Therefore, we decided to use GPH1 tagged with green fluorescent protein

122 (GFP) as a phenotypic readout for experiments aimed at dissecting which variants at the IRA2

123 locus affect the expression of other genes in trans. 


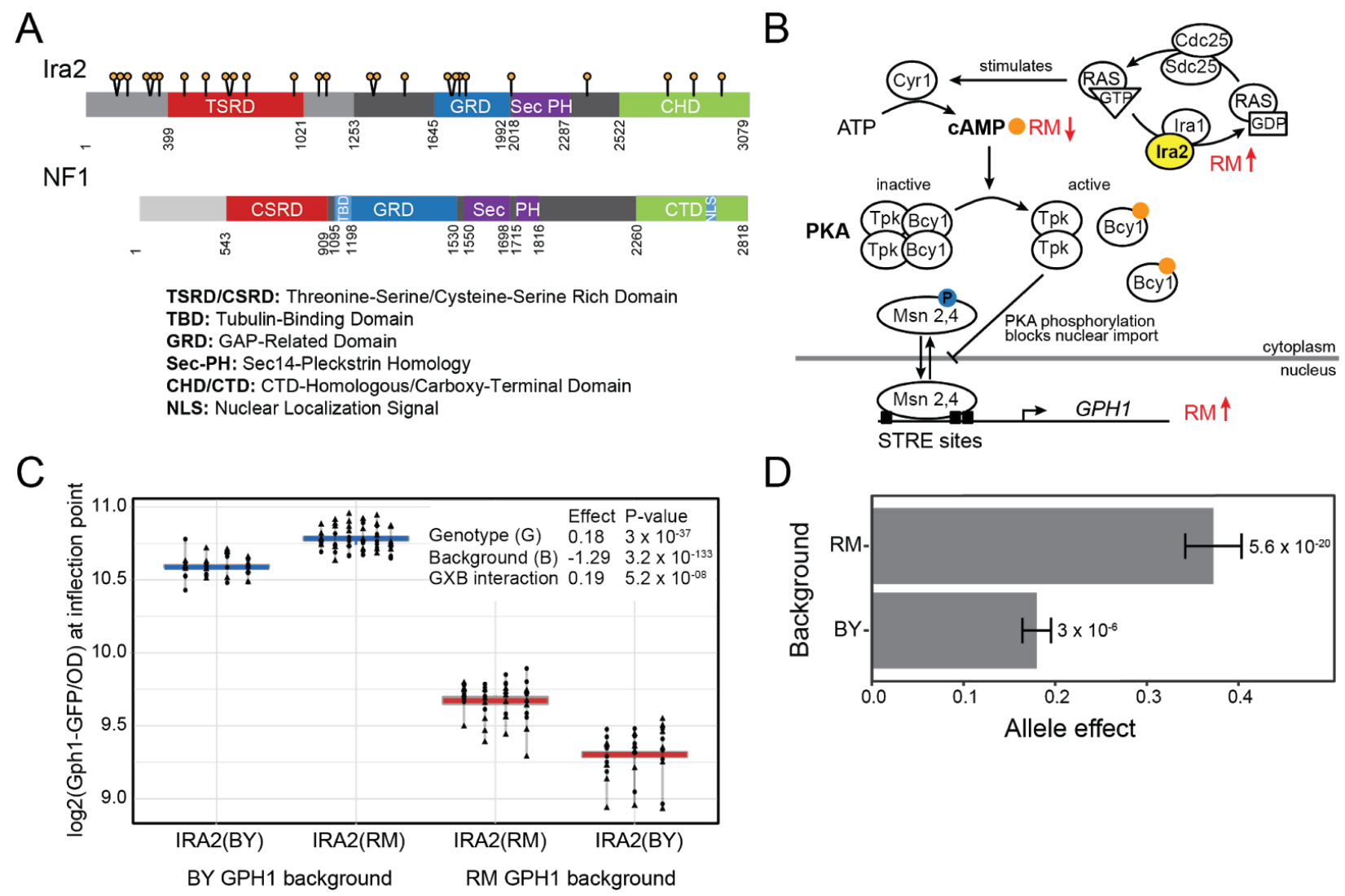

124 Figure 1. RM variants within the coding region of IRA2 increase Gph1-GFP expression in trans. A: Schematic showing nonsynonymous variants (lollipops) and domains in the Ira2 protein. Defined domains are depicted with colored boxes. The matching dark gray region indicates the continuous homologous region between IRA2 and NF1. The NF1 schematic is modified from (Bergoug et al., 2020). Details on domain designations for Ira2 can be found in Materials and Methods. B: The role of IRA2 in the cAMP-PKA pathway including GPH1. C: Measurement of the effect of the IRA2 coding variants in the BY and RM backgrounds. Different shapes represent different plate reader runs. Lines connect measurements of the same transformant. For each strain, $3-6$ independent transformants were phenotyped $10-11$ times during two plate reader runs. The boxplots show the median as thicker central lines and the first and third quartiles computed on averaged phenotypes per transformant. D: The IRA2 allele effect in the BY and RM backgrounds. The allele effect is the $\log _{2}$ of the average Gph1-GFP expression in the presence of the RM allele minus that in the presence of the BY allele. Error bars are the standard error of the estimate. 
138 Table 1 - The 26 nonsynonymous BY/RM variants in IRA2

\begin{tabular}{|c|c|c|c|c|c|c|c|c|c|c|c|}
\hline Variant & Block & $\begin{array}{l}\text { Location } \\
\text { on chr } \\
\text { XV }\end{array}$ & $\begin{array}{l}\text { BY/RM } \\
\text { codons }^{1}\end{array}$ & $\begin{array}{l}\text { BY/RM } \\
\text { amino acids } \\
\text { \& position }\end{array}$ & $\begin{array}{l}\text { Taiwanese } \\
\text { variant }\end{array}$ & $\begin{array}{l}\text { S. } \\
\text { Paradoxus } \\
\text { variant }\end{array}$ & $\begin{array}{l}\text { Derived } \\
\text { allele } \\
\text { frequency }\end{array}$ & $\begin{array}{l}\text { Derived } \\
\text { allele } \\
\text { PROVEAN } \\
\text { prediction }\end{array}$ & $\begin{array}{l}\text { Derived } \\
\text { allele } \\
\text { PROVEAN } \\
\text { score }\end{array}$ & $\begin{array}{l}\text { RM } \\
\text { allele } \\
\text { effect }\end{array}$ & $p$-value \\
\hline 1 & 1 & 171511 & acC/acT & $\mathrm{N} 148 \mathrm{H}$ & BY & BY & 0.53 & Neutral & -1.23 & -0.01 & 0.6 \\
\hline 2 & 1 & 171515 & Aac/Cac & S149N & RM & BY & 0.35 & Neutral & -0.105 & -0.017 & 0.35 \\
\hline 3 & 1 & 171671 & aGt/aAt & N201S & BY & BY & 0.18 & Neutral & -1.94 & 0 & 0.99 \\
\hline 4 & 1 & 171973 & Tat/Cat & Y302H & BY & BY & 0.52 & Deleterious & -3.12 & 0.016 & 0.32 \\
\hline 5 & 1 & 171985 & Cat/Gat & H306D & BY & BY & 0.52 & Neutral & 0.3 & -0.01 & 0.58 \\
\hline 6 & 1 & 172102 & Gct/Act & A345T & BY & BY & 0.29 & Neutral & -1.85 & -0.012 & 0.5 \\
\hline 7 & 1 & 172468 & Aat/Gat & N467D & BY & BY & 0.23 & Neutral & -1.24 & -0.011 & 0.57 \\
\hline 8 & 1 & 172768 & Gtc/Atc & V567I & BY & BY & 0.3 & Neutral & -0.07 & 0.008 & 0.6 \\
\hline 9 & 2 & 173080 & Gtg/Atg & V671M & RM & RM & 0.06 & Neutral & -0.15 & 0.009 & 0.66 \\
\hline 10 & 2 & 173105 & aAt/aTt & N679I & BY & BY & 0.5 & Deleterious & -5.64 & -0.027 & 0.17 \\
\hline 11 & 2 & 173340 & $\mathrm{caA} / \mathrm{caC}$ & Q757H & BY & BY & 0.13 & Neutral & -0.73 & 0.043 & 0.057 \\
\hline 12 & 2 & 174019 & Agc/Tgc & S984C & BY & BY & 0.38 & Deleterious & -2.83 & -0.032 & 0.073 \\
\hline 13 & 2 & 174364 & $\mathrm{Ccg} / \mathrm{Tcg}$ & P1099S & RM & RM & 0.42 & Neutral & -2.323 & -0.016 & 0.4 \\
\hline 14 & 2 & 174472 & $\mathrm{Ctt} / \mathrm{Ttt}$ & L1135F & BY & BY & 0.51 & Neutral & -1.85 & 0.049 & 0.0065 \\
\hline 15 & 3 & 175135 & Ata/Gta & I1356V & BY & BY & 0.5 & Neutral & -0.39 & 0.028 & 0.17 \\
\hline 16 & 3 & 175142 & $\mathrm{tCt} / \mathrm{tTt}$ & S1358F & BY & BY & 0.51 & Neutral & 2.53 & -0.034 & 0.066 \\
\hline 17 & 3 & 175588 & Atc/Gtc & I1507V & RM & RM & 0.36 & Neutral & -0.43 & 0.069 & 0.0027 \\
\hline 18 & 3 & 176239 & Gca/Tca & A1724S & RM & RM & 0.41 & Neutral & -1.057 & -0.034 & 0.06 \\
\hline 19 & 3 & 176268 & aaA/aaT & K1733N & BY & BY & 0.6 & Neutral & 0.56 & 0.026 & 0.163 \\
\hline 20 & 3 & 176348 & aAc/aGc & N1760S & RM & BY & 0.33 & Neutral & 0.653 & -0.007 & 0.69 \\
\hline 21 & 3 & 176441 & $\mathrm{tCa} / \mathrm{tTa}$ & S1791L & BY & BY & 0.52 & Neutral & -1.65 & -0.003 & 0.89 \\
\hline 22 & 4 & 177067 & $T t t / G t t$ & F2000V & BY & BY & 0.39 & Neutral & -0.38 & -0.066 & 0.00047 \\
\hline 23 & 4 & 178159 & $\mathrm{Ccc} / \mathrm{Tcc}$ & P2364S & BY & BY & 0.41 & Deleterious & -4.08 & 0.042 & 0.02 \\
\hline 24 & 4 & 179291 & $\mathrm{gCc} / \mathrm{gTc}$ & A2741V & BY & BY & 0.25 & Neutral & 0.88 & 0.006 & 0.74 \\
\hline 25 & 4 & 179652 & $\mathrm{ttT} / \mathrm{ttG}$ & F2861L & BY & BY & 0.1 & Neutral & 2.56 & 0.006 & 0.74 \\
\hline 26 & 4 & 180056 & aAt/aCt & N2996T & RM & RM & 0.23 & Neutral & 0.072 & 0.009 & 0.62 \\
\hline
\end{tabular}

$139{ }^{1}$ The base in the codon altered by the variant is indicated by a capital letter.

140 To test if IRA2 is indeed the causal gene at this locus, we exchanged alleles of the IRA2 ORF in

141 strains carrying Gph1-GFP using our CRISPR-Swap strategy for rapid genome engineering

142 (Lutz et al., 2019). We then measured Gph1-GFP fluorescence and optical density $\left(\mathrm{OD}_{600}\right)$ of the

143 engineered strains during 24 hours of growth in a plate reader. The Gph1-GFP expression level

144 was extracted at the inflection point of the growth curve and normalized using the $\mathrm{OD}_{600}$ value at

145 this time (see Materials and Methods). The presence of the RM allele compared to the BY allele

146 resulted in significantly higher Gph1-GFP expression, confirming that coding variants in IRA2

147 contribute to the effects of this locus (Figure 1C). The effect was present in both the BY and RM 
148 strain backgrounds. However, the effect was larger in RM than in BY (Analysis of variance [ANOVA], p-value for the interaction between IRA2 genotype and strain background: $5 \times 10^{-8}$; Figure 1C and D; and Table S3), suggesting the presence of epistatic interactions between variation in the IRA2 coding region and other variants elsewhere in the genome.

152 Increased expression of Gph1-GFP in the presence of the IRA2-RM allele is consistent with the 153 direction of known effects of this locus on GPH1 mRNA and protein (Albert et al., 2018, 2014).

154 Based on the function of Ira2 (Figure 1B), increased GPH1 expression is expected in the 155 presence of a more active IRA2 allele. Thus, in accordance with previous results (Smith and 156 Kruglyak, 2008), our findings suggest that the RM allele of IRA2 has higher activity than the BY 157 allele.

\section{$I R A 2$ harbors multiple causal variants that show epistatic interactions}

To narrow in on the causal variant in the large IRA2 gene, we divided the IRA2 ORF into four blocks balancing size and number of nonsynonymous variants (Figure 2A). We performed these experiments in BY due to the higher efficiency of genome engineering in this strain background (Lutz et al., 2019). We created 16 chimeric alleles representing all combinations of the four blocks to also allow testing for non-additive ("epistatic") interactions between the blocks. Each block combination was represented by five or six independent transformants that were each assayed five times for their effect on Gph1-GFP expression.

At each of the first three blocks, the IRA2 RM sequence significantly increased Gph1-GFP expression, with the largest effect resulting from block 1 (Figure 2B; Table 2; Figure S1 and Table S4). Thus, there must be at least three causal variants in the IRA2 RM allele, with at least one variant in each of blocks 1,2 and 3 .

We noticed that one combination of blocks (with the RM at the first 3 blocks and the BY allele at the last block, "RRRB") resulted in significantly higher Gph1-GFP expression than the allele carrying RM alleles at all four blocks (One-way ANOVA: $p=0.035$, Figure 2B). Thus, higher Gph1-GFP levels than those driven by the full RM allele can be achieved by combinations of BY and RM variants. Considering that block 4 had no effect when swapped in isolation (Figure 2B; Table 2 and Table S4), this difference between the RRRB construct and the full RM allele also suggests that there are epistatic interactions between variants in block 4 and variants in the other blocks.

To comprehensively test for epistatic interactions among blocks, we performed ANOVA on our fully crossed set of block chimeras. Three combinations of blocks showed nominally significant non-additive interactions (Table 2), which exceeds the expectation of 0.6 out of 12 interaction tests under a null model without epistasis. The strongest interaction was seen between block 1 and block 2 and is visualized in Figure 2C. In all three significant interactions, the observed 
183 Gph1-GFP expression driven by the combined blocks was less than that expected from the sum 184 of the individual block effects (note negative effect signs in Table 2), indicating negative

185 epistasis between these blocks of variants. In summary, IRA2 harbors multiple variants that affect

186 Gph1-GFP expression in trans, some of which interact in a non-additive manner.

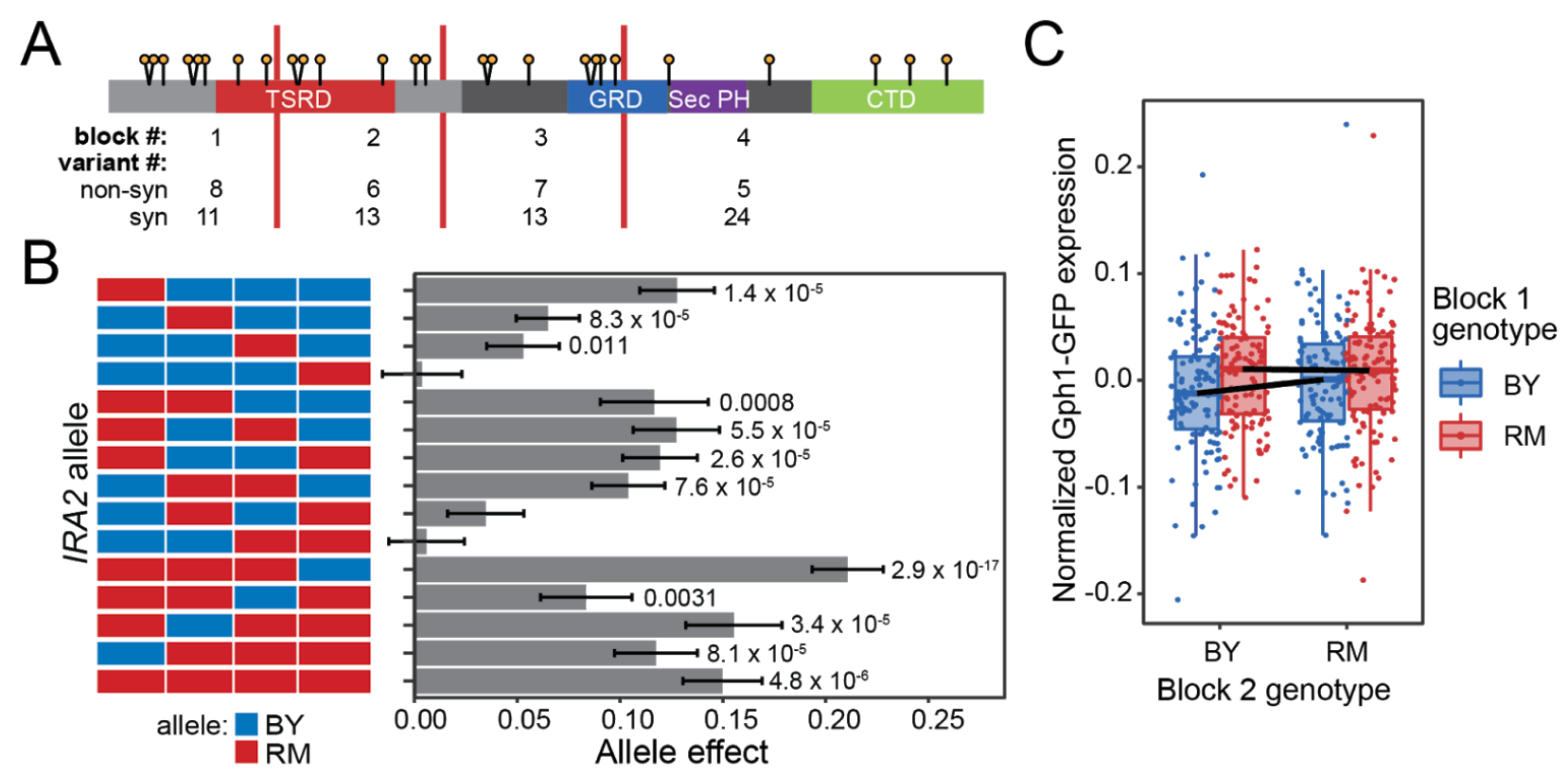

187 Figure 2: Effect of blocks of IRA2 variants on Gph1-GFP expression. A: Schematic of Ira2 showing the nonsynonymous variants (lollipops) and block junctions (red vertical lines). B: Allele effect of each of the sixteen block combinations compared to an allele with BY in all blocks. P-values are for a comparison between the given allele and the BY allele, only significant values are displayed. Error bars are standard errors of the given estimate. C: Epistasis between blocks 1 and 2. The figure shows residual Gph1-GFP expression after removal of random effects of plate and transformant identity (Materials and Methods). Measurements are grouped by their alleles at blocks 1 and 2. The remaining two blocks are any combination of BY or RM. The horizontal black lines connect the average allele effect for the indicated genotype. Epistasis is highlighted by the steeper slope of the line when block 1 is BY rather than RM. 
197 Table 2 - ANOVA of the effects of blocks 1 to $\mathbf{4}$ and all possible interaction terms

\begin{tabular}{|c|c|c|c|c|}
\hline & Effect & $\begin{array}{l}95 \% \text { effect } \\
\text { confidence } \\
\text { interval }\end{array}$ & F-value & p-value \\
\hline b1 & 0.13 & $0.08-0.19$ & 85.57 & $3.9 \times 10^{-14}$ \\
\hline b2 & 0.06 & $0.01-0.12$ & 16.86 & $9.9 \times 10^{-5}$ \\
\hline b3 & 0.05 & $0.001-0.11$ & 21.93 & $1.2 \times 10^{-5}$ \\
\hline b4 & 0.004 & $-0.05-0.06$ & 2.96 & 0.09 \\
\hline b1:b2 & -0.08 & $-0.16--0.004$ & 8.51 & 0.005 \\
\hline b1:b3 & -0.05 & $-0.13-0.03$ & 0.04 & 0.84 \\
\hline b1:b4 & -0.01 & $-0.10-0.07$ & 0.04 & 0.85 \\
\hline b2:b3 & -0.01 & $-0.08-0.05$ & 6.34 & 0.01 \\
\hline b2:b4 & -0.03 & $-0.11-0.05$ & 1.16 & 0.28 \\
\hline b3:b4 & -0.005 & $-0.12-0.03$ & 0.00 & 0.97 \\
\hline b1:b2:b3 & 0.1 & $-0.002-0.22$ & 0.66 & 0.42 \\
\hline b1:b2:b4 & 0.009 & $-0.11-0.12$ & 3.36 & 0.07 \\
\hline b1:b3:b4 & 0.09 & $-0.03-0.20$ & 0.05 & 0.82 \\
\hline b2:b3:b4 & 0.09 & $-0.004-0.19$ & 0.18 & 0.67 \\
\hline b1:b2:b3:b4 & -0.16 & $-0.32-0.0002$ & 4.22 & 0.04 \\
\hline
\end{tabular}

Effect estimates are polarized such that positive values correspond to higher expression linked

199 to the respective RM allele. Effects were computed using a mixed-effects linear model and

200 p-values computed by type I ANOVA. Effect size confidence intervals were estimated using a

201 bootstrap procedure. The displayed p-values were not corrected for multiple testing. P-values

202 less than 0.05 are shown in bold.

\section{The effect of each nonsynonymous RM variant is small compared to the full RM allele}

204 To examine the effects of single variants in IRA2 on Gph1-GFP expression, we engineered 26

205 IRA2 strains. Each strain carried the RM allele at a single nonsynonymous variant resulting in a

206 single amino acid change, with all other variants carrying the BY allele. We measured the effect

207 of each single variant allele on Gph1-GFP expression (Figure 3A; Figure S2; and Table S5). 
A

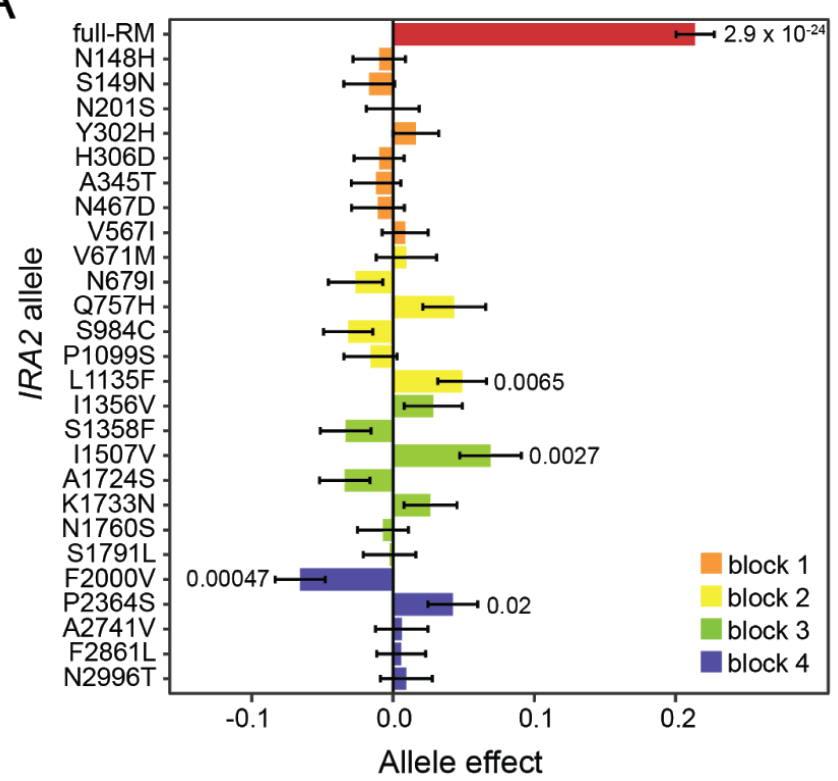

B

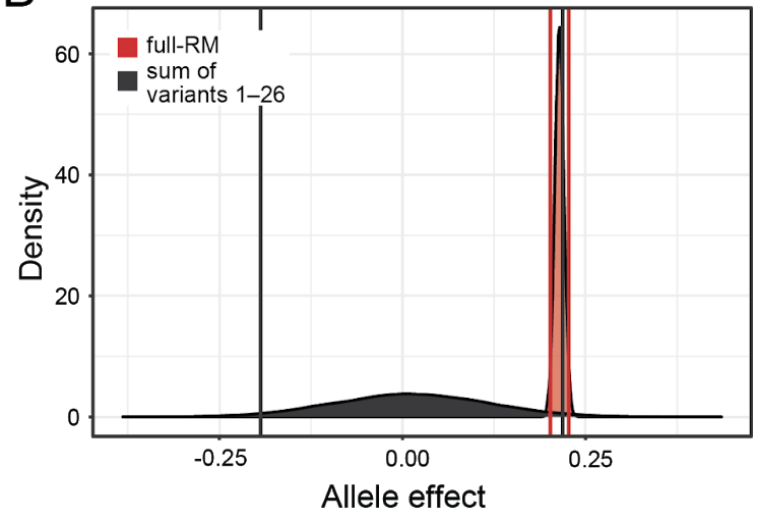

209 Figure 3: Analysis of the effects of the nonsynonymous variants in IRA2. A: Effect of each nonsynonymous RM variant allele on Gph1-GFP expression. Each strain was represented by nine transformants that were each measured five times. The first eight variants $(\mathrm{N} 148 \mathrm{H}$ through V567I) were measured three additional times in an additional plate configuration. P-values are

213 shown for a comparison between the given allele and the BY allele. Only significant p-values

214 less than 0.05 are displayed. B: Bootstrap analysis of the effect of the full RM allele compared 215 to the summed effects of the twenty-six variants. The same-colored vertical lines mark the 216 central $95 \%$ quantile range for each bootstrap distribution.

217 Four of the single-variant RM alleles (L1135F, I1507V, F2000V, and P2364S) resulted in

218 Gph1-GFP expression significantly different from the BY allele. Three of these causal variants

219 were predicted to have a neutral effect by PROVEAN, while the fourth, P2364S, was predicted

220 to be deleterious (Table 1). None of the four variants were rare in the population, with derived

221 allele frequencies ranging from 0.36 to 0.51 . The causal variants are located in three different

222 blocks: L1135F is in block 2, I1507V is in block 3, and F2000V and P2364S are in block 4.

223 Alignment of IRA2 with its human homolog NF1 revealed that L1135F is in a region that does

224 not have homology with NF1, while I1507, F2000V and P2364S are in regions that are

225 conserved with humans (Figure 1). While three of the single RM variants increased Gph1-GFP

226 expression, the F2000V variant reduced it. This direction of effect of F2000V is in the opposite

227 direction of that of the full IRA2-RM allele, revealing transgressive segregation of causal variants

228 in a single gene. The effect of F2000V is likely canceled out by its neighboring variant P2364S,

229 at which the RM allele significantly increased Gph1-GFP. The canceling of these variant effects

230 is in agreement with the fact that block 4 as a whole did not significantly alter Gph1-GFP

231 expression (Figure 2B). These results show that no single variant underlies the trans effect of

232 IRA2-RM on Gph1-GFP expression. Instead, a combination of multiple variants must underlie

233 the overall effect of the full RM allele at IRA2. 
234 The effects of the single variants were small compared to the effect of the entire IRA2-RM allele

235 (Figure 3A). To test whether their joint effect can account for the entire IRA2 effect in an 236 additive manner, we used a bootstrap strategy that accounted for measurement error. We created

23710,000 bootstrap datasets by randomly sampling with replacement from our individual

238 measurements. In each dataset, we computed the observed difference between Gph1-GFP

239 abundance for the IRA2-BY and the full IRA2-RM allele, as well as the sum of the effects driven

240 by each of the 26 single variants. The central $95 \%$ of the resulting bootstrap distributions

241 overlapped, such that we cannot formally rule out that the sum of the single variant effects

242 accounts for the effect of the full RM allele in an additive manner (Figure 3B). However, the

243 overlap was mostly due to the very long tail of the summed single variant distribution, such that

244 only $3.6 \%$ of this distribution exceeded the $2.5 \%$ quantile of the full RM allele effect. As also

245 suggested by the significant epistatic interactions among the four blocks above, it remains

246 possible that epistatic interactions among variants across the length of IRA2 are required to

247 generate the effect of the full IRA2-RM allele.

\section{Multiple, epistatic causal variants are near the 5'-end of IRA2}

249 The results above suggest considerable complexity of genetic variation within IRA2, with

250 multiple causal variants and non-additive interactions between different regions of the ORF. To

251 dissect this architecture further, we focused on block 1. Although block 1 had the largest effect

252 on Gph1-GFP expression, none of the eight single variants in this block had a significant effect

253 on their own. The variants in block 1 could have real but subtle effects, such that their combined

254 effect could account for the block 1 effect even if no individual variant reached statistical

255 significance. However, bootstrap analysis showed that the additive effects of these variants

256 cannot account for the effect of block 1 (Figure 4A), suggesting a non-additive interaction

257 between at least two of the eight variants within block 1 .

258 We further divided block 1 into sub-block 1, containing the first five nonsynonymous variants

259 and assayed its effect on Gph1-GFP expression (Figure 4B; Figure S3 and Table S6). The

260 effect of sub-block 1 was significant $\left(p=4 \times 10^{-7}\right)$, but was smaller than the entire effect of block

$2611(\mathrm{p}=0.05)$. This result suggests that the effect of block 1 is caused by multiple variants: at least

262 one among the first five variants and at least one among the following three variants.

263 Furthermore, bootstrap analysis showed that the summed effects of the first five variants are

264 unlikely to account for the effect of sub-block 1 (Figure 4C). Thus, further division of block 1

265 caused its effects to splinter and uncovered the existence of non-additive interactions among the

266 five variants closest to the 5'-end of the gene. 
A

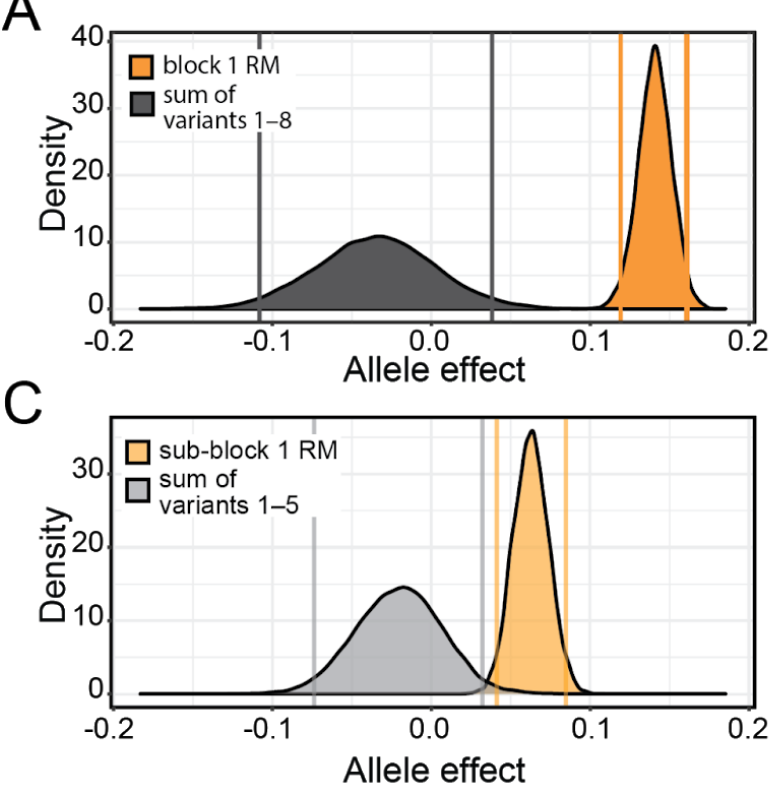

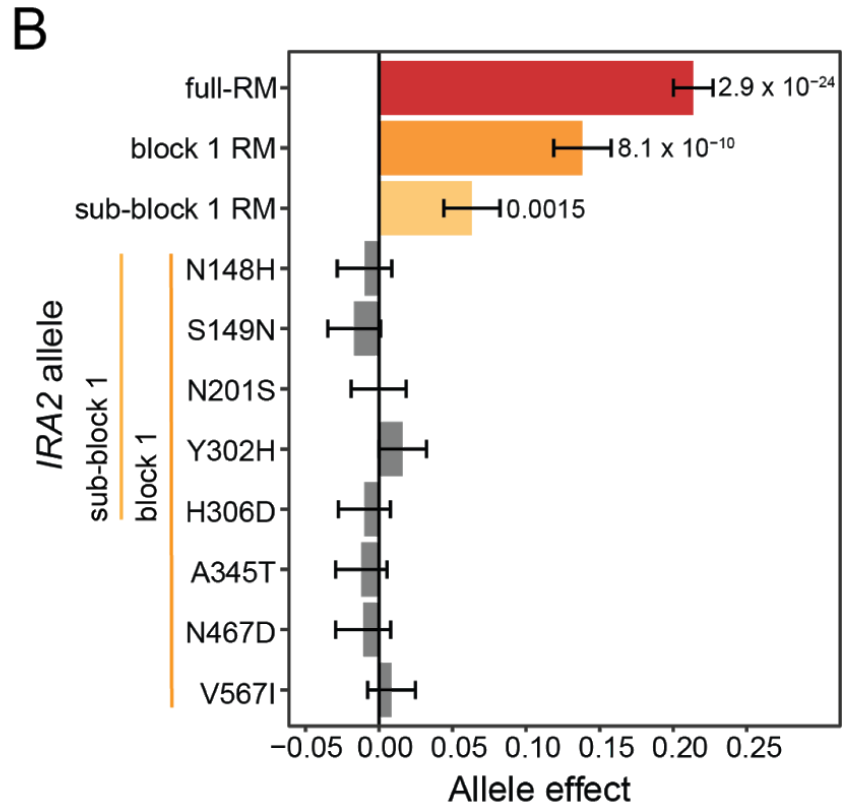

267 Figure 4: Analysis of the effects of the variants in block 1 and sub-block 1. A: Bootstrap analysis of the effect of the block $1 \mathrm{RM}$ allele compared to the summed effects of the eight variants in block 1 . The same-colored vertical lines mark the central $95 \%$ quantile range for each distribution. B: Allele effects of the designated IRA2 genotypes. P-values are for a comparison between the given allele and the BY allele. Only significant p-values less than 0.05 are displayed. Each strain was represented by nine or ten transformants that were each phenotyped three (block 1 RM and sub-block 1 RM) or eight (variants 1-8) times. C: Bootstrap analysis of the effect of the sub-block $1 \mathrm{RM}$ allele compared to the summed effects of the five variants in sub-block 1.

\section{No evidence for an effect of synonymous variants in block 1}

277 Instead of epistasis among nonsynonymous variants, a possible alternative explanation for the 278 differences between the large effects we measured for the blocks and the smaller additive effects 279 of their single nonsynonymous variants could be effects caused by the synonymous variants 280 within the blocks. Specifically, the engineered blocks were generated by amplifying the block regions from BY and RM genomic DNA. Therefore, the blocks include synonymous variants

282 that are not present in our single-variant experiments. Although they do not change protein

283 sequences, synonymous variants have been reported to affect complex traits (Sharon et al., 2018;

284 She and Jarosz, 2018). For example, their effects could arise from changes in translation that 285 could affect Ira2 expression or function, especially for variants close to the 5'-end of the gene 286 (Plotkin and Kudla, 2011; Tuller et al., 2010). We chose to test the combined effect of the eleven 287 synonymous variants in block 1. Block 1 had the largest effect of the four blocks, while none of 288 its eight nonsynonymous variants had a significant effect on their own. We engineered alleles of 289 block 1 that carried each of the four different combinations of the synonymous variants and 
290 nonsynonymous variants. These alleles showed a strong effect of the nonsynonymous variants in block 1 (ANOVA $\left.p=8 \times 10^{-7}\right)$. The synonymous variants had no discernible effect $(p=0.20$;

292 Figure 5). Thus, the overall effect of block 1 must be due to multiple nonsynonymous variants

293 with non-additive interactions.

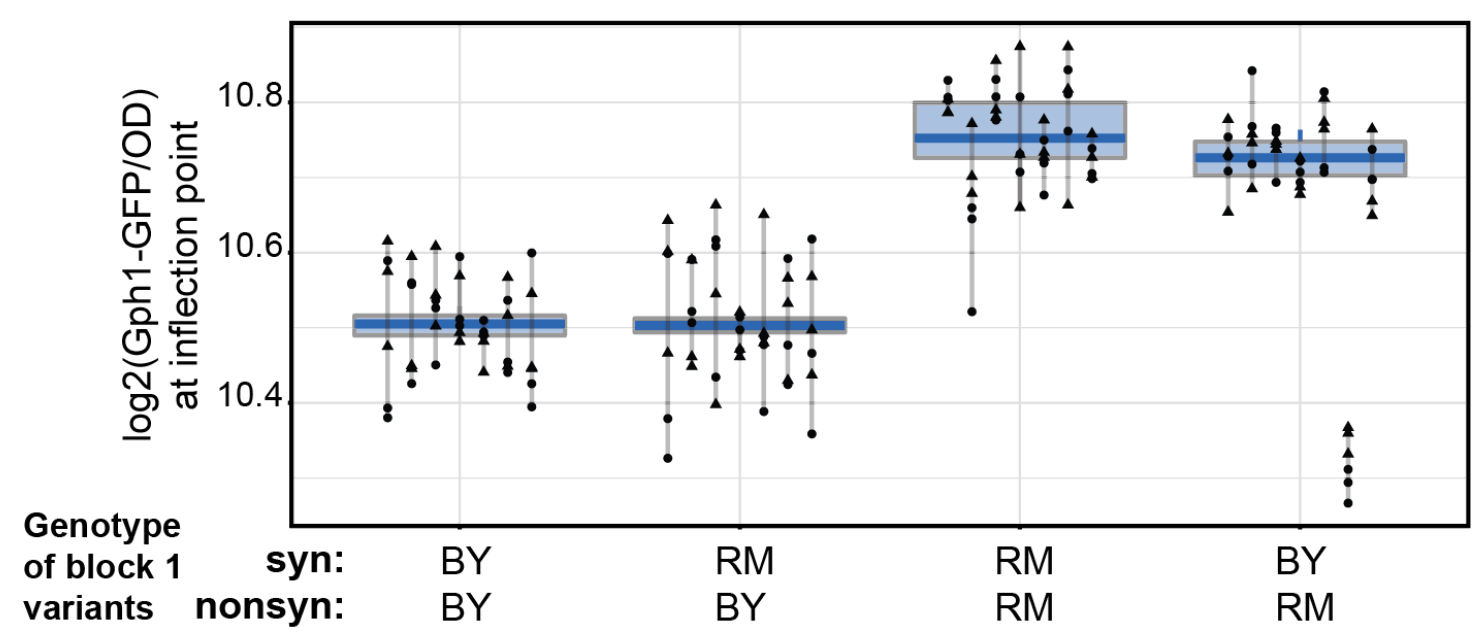

294 Figure 5: Measurement of the effect of the nonsynonymous variants in block 1. Different shapes represent different plate reader runs. Lines connect measurements of the same transformant. For each strain, seven independent transformants were phenotyped six times. The boxplots show the median as thicker central lines and the first and third quartiles computed on averaged phenotypes per transformant.

\section{Discussion}

300 Our fine-mapping experiments in the IRA2 gene revealed multiple causal variants spread 301 throughout the IRA2 ORF that likely act together to create the trans-eQTL hotspot at this gene.

302 Altogether, there must be at least seven causal variants in this gene. In our single-variant 303 experiments, we directly identified one variant each in block 2 and block 3, and two variants in 304 block 4 . In addition, there must be at least three causal variants in block 1, with at least two in 305 sub-block 1, that epistatically interact to create the strong increase in Gph1-GFP expression 306 caused by this block. In addition to this localized epistasis within block 1, which covers a poorly conserved region of the ORF with unknown function, we also found evidence for epistasis between variants spread further across the gene body. Thus, this single gene harbors a complex molecular genetic architecture.

310 We were able to detect the small effects of the causal variants in IRA2 because in conjunction 311 they create a large effect that manifests as a highly pleiotropic trans-eQTL hotspot that drew our 
312 attention to this region. It is interesting to speculate how many variants of similar effect may go

313 unnoticed by QTL mapping because they fail to co-occur in a fashion that creates a detectable

314 QTL (Bernstein et al., 2019; Kroymann and Mitchell-Olds, 2005; Metzger and Wittkopp, 2019).

315 Our work has several limitations. First, we used a single, GFP-tagged gene as a phenotype for

316 fine-mapping trans effects of IRA2. We cannot rule out that other traits affected by the IRA2

317 locus are shaped by different causal variants, possibly in genes close to IRA2. However, we deem

318 it unlikely that traits that are truly influenced by the $I R A 2$ gene are shaped by different variants

319 within IRA2, given that Ira2 acts high in the RAS signaling pathway (Figure 1B). Assuming that

320 IRA2 variants ultimately affect phenotypes via altered cAMP/PKA signaling, it is unclear how

321 variants could have trait-specific downstream effects. A possible exception may occur if Ira2

322 performs unknown functions other than regulating RAS. Second, while we have provided

323 evidence for the existence of epistatic interactions between variants in IRA2, we have not

324 determined the specific variants that engage in these interactions. Because epistatic interactions

325 are probably required for explaining the full difference between the BY and RM alleles, the

326 precise molecular basis for this strain difference remains open.

327 In conclusion, our result of multiple causal variants in the IRA2 gene mirrors observations about

328 genetic architectures at coarser scales. Quantitative traits including gene expression can be

329 highly polygenic (Bloom et al., 2013; Boyle et al., 2017; Flint and Mackay, 2009; Visscher et al.,

330 2017), and the expression of a given gene is shaped by many loci across the genome (Albert et

331 al., 2018; Brion et al., 2020; Metzger and Wittkopp, 2019). Each of these loci can harbor

332 multiple causal genes (Bernstein et al., 2019; Steinmetz et al., 2002). In turn, these genes can

333 have multiple causal variants, extending the pattern of multifactorial complexity to the finest

334 possible scale of individual nucleotides in a single gene. It remains to be explored whether and

335 why certain genes are more prone to carrying single, strong variants versus multiple variants

336 with smaller effect. 


\section{Materials and Methods}

\section{Yeast strains, primers, and media}

339 The $S$. cerevisiae strains used here are derived from S288C (BY4741 (MATa, his3 $\Delta 1$ leu2 $\Delta 0$

340 met15 $\Delta 0$ ura3 $\Delta 0$ ), referred to as "BY" in the text) and RM-11a, (RM HO(BY) (MATa,

341 his3 $\Delta 1::$ CloNAT, leu2 $\Delta 0$, ura3 $\Delta 0 \mathrm{HO}(\mathrm{BY}$ allele) AMN1(BY allele), referred to as "RM"). A

342 complete listing of the strains used in this study can be found in Supplementary Table 1. All

343 primers are listed in Supplementary Table 2.

344 We used the following media (recipes are for 1L):

345 For general yeast strain growth and storage:

346 YPD (10 g yeast extract, $20 \mathrm{~g}$ peptone, $20 \mathrm{~g}$ glucose)

347 For selection of the GFP-HIS3MX6 cassette in GPH1 tagging:

348 SDC-His (1.66 g SC -His -Leu -Ura, $100 \mathrm{mg}$ leucine, $200 \mathrm{mg}$ uracil, $20 \mathrm{~g}$ glucose)

349 For selection of the KanMX4 cassette in IRA2 gene deletions and verifying allele exchanges by

350 CRISPR-Swap:

351 YPD +G418 (G418 sulfate (Fisher Scientific cat\# BP6731); $200 \mu \mathrm{g} / \mathrm{ml}$ )

352 For selecting for the CRISPR-Swap plasmid in cells after transformation:

353 SDC-Leu (1.66 g SC -His -Leu -Ura (Sunrise Science; cat\# 1327-030), 50 mg histidine, 200 mg

354 uracil, $20 \mathrm{~g}$ glucose)

355 For phenotyping Gph1-GFP expression:

356 YNB LowFlo (6.7 g yeast nitrogen base -folic acid -riboflavin with ammonium sulfate and

357 without amino acids (Sunrise Science; cat\# 1536-050), 20 g glucose, $50 \mathrm{mg}$ histidine, $100 \mathrm{mg}$

358 leucine, $50 \mathrm{mg}$ methionine, $200 \mathrm{mg}$ uracil). Sterilized by filtration.

359 For iodine staining:

360 YNB (6.7 g yeast nitrogen base with ammonium sulfate and without amino acids (BD

361 Biosciences cat\# 291940), $20 \mathrm{~g}$ glucose, $50 \mathrm{mg}$ histidine, $100 \mathrm{mg}$ leucine, $50 \mathrm{mg}$ methionine, 200

362 mg uracil)

363 For solid media, $20 \mathrm{~g} / \mathrm{L}$ agar was added prior to autoclaving. Yeast were grown at $30^{\circ} \mathrm{C}$. 


\section{GPH1-GFP tagging and IRA2 gene deletions}

365 Insertions of cassettes for genome modification were performed using a standard PCR-based 366 one-step method (Longtine et al., 1998). Yeast transformations were performed using a standard 367 LiAc procedure (Gietz and Schiestl, 2007). Transformants expressing the selectable marker were 368 single colony purified and insertion of the cassette into the correct location and absence of the 369 unmodified wildtype allele were verified by colony PCR.

393 The IRA2-block alleles were created using gene splicing by overlap extension (SOEing) (Horton 394 et al., 1989). Each of the four block regions was PCR amplified from BY (BY4741 for blocks 1

Strain BY GPH1-GFP:HIS3MX6 (YFA0644) was obtained from the GFP collection (Huh et al., 2003). RM HO(BY) GPH1-GFP:HIS3MX6 (YFA0649) was created as follows: the GPH1-GFP allele was PCR amplified from YFA0644 genomic DNA using primers OFA0471 and OFA0472 and integrated into RM HO(BY) (YFA0254).

\section{Strains BY GPH1-GFP ira2A:KanMX (YFA0650 and YFA01430) and RM GPH1-GFP} ira2::KanMX (YFA0654) were created by integration of the ira2 $\Delta:: k a n M X$ allele, which was PCR amplified from YFA0666 genomic DNA using primers OFA0468 and OFA0469. BY GPH1-GFP ira2 $\Delta: \mathrm{KanMX}$ was created twice because glycerol stock storage at $-80^{\circ} \mathrm{C}$ of YFA0650 resulted in a stark reduction in CRISPR-Swap efficiency.

Strain BY GPH1-GFP ira2_block1 $\Delta:$ :KanMX (YFA1443) was created by replacing IRA2 block 1 sequence with the KanMX cassette PCR amplified from nej1 $\Delta:$ KanMX (YFA0007) genomic DNA using primers OFA1079 and OFA1080.

\section{Creation of $I R A 2$ allele fragments used as repair templates for CRISPR-Swap}

All IRA2 allele fragments were PCR amplified from genomic DNA or commercially synthesized DNA using Phusion Hot Start Flex DNA polymerase (NEB). Genomic DNA was isolated using the ten-minute preparation (Hoffman and Winston, 1987). All PCR fragments were analyzed by agarose gel electrophoresis, excised, purified using Monarch DNA Gel Extraction Kit (NEB), and quantified using Qubit fluorometric quantification (Thermo Fisher Scientific).

The IRA2(BY) allele was amplified from BY4741 or YLK1879 genomic DNA and the IRA2(RM) allele (sometimes referred to as "full-RM") from YFA0254 or YLK1950 genomic DNA using primers OFA0468 and OFA0469. These primers amplify a 9,431 bp IRA2 fragment with termini (123 bp at the 5' end and $68 \mathrm{bp}$ at the 3' end) that have identical sequence to the region flanking the ira2s::KanMX cassette. and 2 and YLK 1879 for blocks 3 and 4) and RM (YFA0254) genomic DNA. Primers used were: block 1, OFA0467 and OFA0455; block 2, OFA0456 and OFA0457; block 3, OFA0458 and OFA0459; and block 4, OFA0460 and OFA0544. Pairwise fusions of block 1 and block 2 (4 
combinations) were amplified with primers OFA0467 and OFA0457 and block 2 and block 3 (4 combinations) were amplified with primers OFA0458 and OFA0544. Finally, all pairwise fusions of block 1 and 2, and block 3 and 4 (16 combinations) were fused by amplification with primers OFA0468 and OFA0469.

Single-variant alleles were created by PCR SOEing using complementary primers containing the desired RM variant (see Table S2). In the first step, two PCR amplifications were performed using BY (YLK1879) genomic DNA as a template. The first amplification used the variant-specific reverse primer and OFA0467, and the second amplification used the variant-specific forward primer and OFA0544. In the second step, the two PCR fragments were fused using primers OFA0468 and OFA0469.

The IRA2 block1 RM allele was created as described above for the IRA2-block alleles. The IRA2 sub-block 1 RM allele was created by PCR SOEing of two PCR fragments. Fragment 1 was amplified with primers OFA0467 and OFA0589 from RM genomic DNA (YLK1950), and fragment 2 was amplified with primers OFA0536 and OFA0544 from BY genomic DNA (YLK1879). The two PCR fragments were fused using primers OFA0468 and OFA0469.

The IRA2-block 1 synonymous and nonsynonymous variant alleles were PCR amplified using primers OFA0468 and OFA1124. Amplification with these primers creates a 1,978 bp IRA2 fragment with termini ( $123 \mathrm{bp}$ at the 5 ' end and $54 \mathrm{bp}$ at the 3 ' end) that are identical to the region flanking the ira2_block1 $\Delta::$ KanMX cassette. The synBYnonsynBY and synRMnonsynRM alleles were amplified from BY and RM genomic DNA (YLK1879 and YLK1950, respectively) and the synBYnonsynRM and synRMnonsynBY alleles were amplified from synthetic DNA (Twist Bioscience).

\section{CRISPR-Swap}

We followed the protocol of (Lutz et al., 2019). For each CRISPR-Swap transformation, the amount of the IRA2 allele repair fragments ranged from 1000-1500 ng, and the amount of plasmid pFA0055 (Addgene \#131774) was 330 ng. pFA0055 expresses Cas9 as well as the guide RNA (gCASS5a) that directs Cas9 to the 5'-end of the KanMX cassette. We recovered 10 to 320 transformants when engineering in BY using the 9,431-kb IRA2 repair template and 504 to more than 900 transformants when using the $1,978 \mathrm{~kb} I R A 2$ block 1 repair template. Of single colony streaked transformants, $98-100 \%$ had lost G418 resistance, indicating a successful allele exchange.

The sixteen chimeric block strains were engineered in one batch, as were the strains with the four arrangements of synonymous and nonsynonymous variants in block 1 . The BY and RM background strains were engineered in two batches: one created the BY GPH1-GFP IRA2(BY) and RM GPH1-GFP IRA2(RM) strains and the other the BY GPH1-GFP IRA2(RM) and RM 
433 GPH1-GFP IRA2(BY) strains. The IRA2 single-variant strains were engineered in four batches, 434 corresponding to the four IRA2 blocks. In each batch, a new IRA2(BY) strain was also 435 engineered, and in batches 1, 2 and 4 a new IRA2(RM) strain was engineered. The IRA2(BY) 436 strains from these four batches were similar in their effect on Gph1-GFP expression and 437 therefore were grouped into one IRA2(BY) genotype that served as the wildtype baseline for 438 determining the effects of the other alleles. The IRA2(RM) strains were similarly grouped. The 439 IRA2 block1 RM strain and the IRA2 sub-block 1 RM strain were created in the same batch 440 (block1) as variants $1-8$.

\section{Verification of $\boldsymbol{I R} \boldsymbol{A} 2$ alleles after CRISPR-Swap}

442 All G418 sensitive transformants after CRISPR-Swap were assumed to have exchanged the 443 KanMX allele for the provided IRA2 allele. To verify the presence of the correct IRA2 allele and 444 that strain mix-ups did not occur during the experimental procedures, some of the strains were 445 partially genotyped at different stages (see below for details). Verified strains are designated in 446 Supplementary Table 1.

447 The IRA2 alleles in the BY GPH1-GFP IRA2(BY) (YFA0658 and YFA0659) and RM 448 GPH1-GFP IRA2(RM) (YFA0662 and YFA0663) strains were genotyped as BY or RM based on 449 an EcoRI site present in the RM but not the BY allele. Genomic DNA isolated from these strains 450 was PCR-amplified with OFA0068 and OFA0070. The PCR product was then digested with 451 EcoRI and analyzed by agarose gel electrophoresis.

452 A representative strain, either transformant \#1 or \#2, from each of the sixteen IRA2 block alleles 453 was confirmed to have the expected arrangement of blocks by verification of the expected 454 variants at the block borders after Sanger sequencing. Genomic DNA was isolated from cultures 455 that were started from glycerol stock plates (YPFA010 and YPFA011) and used for PCR 456 amplification. For each strain, the junction between blocks 1 and 2 was amplified using primers 457 OFA0585 and OFA0905 and sequenced with OFA0904, and the junction between blocks 3 and 4 458 was amplified using primers OFA0906 and OFA0907 and sequenced with OFA0903. Prior to 459 sequencing, the fragments were analyzed by gel electrophoresis and purified using a Monarch 460 DNA Gel Extraction Kit (NEB). All strains had the expected variants in the sequenced fragment. 461 One strain, RRBR \#1 had a de novo variant in the sequenced region resulting in an aGc to aTc 462 (S1860I) amino acid change. This strain was not removed from phenotyping and its Gph1-GFP 463 expression was similar to that of the other RRBR transformants. None of the RBRR 464 transformants were sequenced.

465 To identify potential ira2 loss of function alleles among the transformants of the sixteen different 466 IRA2 block strains, cells were spotted onto YNB agar plates and stained with iodine vapors. 
467 Iodine stains wildtype yeast cells containing glycogen a dark reddish brown. Cells with elevated

468 RAS-GTP levels, as in the case of an ira2 $\Delta$, are unable to store glycogen and therefore stain pale 469 yellow in the presence of iodine (Gil and Seeling, 1999). For each of the sixteen strains, $7-8$

470 transformants were stained with iodine and 7 / 127 stained pale yellow, possibly due to errors

471 created during the multiple-round PCR amplification of the IRA2 alleles. Transformants that

472 stained pale yellow were excluded from phenotyping.

473 In the IRA2 single-variant strains, the expected amino acid change was verified for transformants 474 of N148H, S149N, N201S, Y302H, H306D, A345T, N467D, V567I, Q757H, and 1507V.

475 Genomic DNA was isolated from cells taken from the randomly arrayed starter culture plates and 476 PCR amplified using primers OFA1053 and OFA1054. The PCR fragment was then Sanger

477 sequenced in the region of the expected variant using the following primers: OFA0592 for 478 N148H and S149N; OFA0589 for N201S; OFA0591 for Y302H, H306D, and A345T; OFA0536 479 for N467D; OFA0904 for V567I; OFA0539 for Q757H; and OFA0540 for I1507V.

\section{Phenotyping of Gph1-GFP expression in the plate reader}

481 Starter cultures were inoculated with cells from glycerol stocks and grown overnight at $30^{\circ} \mathrm{C}$ in $482800-1000 \mu \mathrm{l}$ of YPD medium in a 2-ml deep-96-well-plate. The plates were sealed with a

483 Breathe Easy membrane (Diversified Biotech) and placed on an Eppendorf Mixmate set at 1100

$484 \mathrm{rpm}$. After overnight growth, the starter culture plates were sealed with aluminum foil and stored 485 at $4^{\circ} \mathrm{C}$ for the duration of phenotyping.

486 For each phenotyping plate run, $10 \mu \mathrm{l}$ of resuspended cells from the starter culture plate was used 487 to inoculate $600-800 \mu \mathrm{l}$ of YNB LowFlo medium in a 2-ml deep-well plate. These pre-cultures 488 were grown overnight as described for the starter cultures. After overnight growth, the 489 pre-cultures were diluted to an $\mathrm{OD}_{600}=0.05$ in $100 \mu \mathrm{l}$ of YNB LowFlo medium in a 96-well flat 490 bottom plate (Costar) and the plates were sealed with a Breathe Easy membrane (Diversified 491 Biotech).

492 The strains were grown for $\sim 24$ hours in a Synergy H1 (BioTek Instruments) plate reader at $30^{\circ} \mathrm{C}$ 493 with readings taken every $15 \mathrm{~min}$ for 97 cycles with $10 \mathrm{sec}$ of orbital shaking between reads and $49411-13$ min between cycles. Cell growth was characterized using absorbance readings at $600 \mathrm{~nm}$ 495 and Gph1-GFP expression was measured from the bottom of the plate using excitation at $488 \mathrm{~nm}$ 496 and emission at $520 \mathrm{~nm}$.

497 Data was processed as described in (Lutz et al., 2019). Briefly, we fit growth curves to each well 498 to identify the inflection point at which the yeast culture begins to exit exponential growth. We 499 extracted the fluorescence and $\mathrm{OD}_{600}$ values at this point as well as the two time points flanking 500 it, took their respective averages, and calculated the $\log _{2}$ of the ratio between the average 

502 well.

504 Conserved regions between Ira2 and NF1 were determined by multiple sequence alignment of

\section{5}

506

fluorescence value and the average $\mathrm{OD}_{600}$ value as the Gph1-GFP expression level for the given

\section{Designation of IRA2 domains}

Ira2/Ira1/NF1 sequences from Candida glabrata (KTB00138.1), Saccharomyces paradoxus (translated from CP020290.1) Kluyveromyces lactis (translated from CR382125.1), Homo sapiens (P21359.2) and Saccharomyces cerevisiae (AAA34710.1 and AAA34709.1) using Clustal Omega (Sievers et al., 2011), as provided through the EMBL-EBI analysis tool API (Madeira et al., 2019).

The CHD (Neurofibromin CTD-homology domain) and Sec-PH (Sec14 homologous and pleckstrin homology-like domain) were defined by previous studies (D'angelo et al., 2006; Luo et al., 2014). We defined here the Threonine-Serine Rich Domain (TSRD). The TSRD extends from amino acid 399 to 1021 and is enriched for serines and threonines, but not enriched for cysteines. This region has 23 / 24 of the detected phosphorylation sites in Ira2 (Holt et al., 2009; Lanz et al., 2021; Swaney et al., 2013). We chose the start of the TSRD at a cluster of serines and the end at the last detected phosphorylated residue. The TSRD is $16.5 \%$ serine and $10.6 \%$ threonine, while the flanking amino acids $1-398$ and $1022-1645$ have $13.1 \%$ and $6.3 \%$ serine and $8.5 \%$ and $5.3 \%$ threonine residues, respectively.

\section{Ancestral alleles and BY and RM allele frequencies}

Ancestral alleles were determined by comparison to two different evolutionary outgroups to BY and RM. First, we determined the nucleotide allele present in the closely related species $S$. paradoxus after an alignment of IRA2(BY) to GenBank \#AABY01000044.1. Second, we obtained the nucleotide allele present in the Taiwanese $S$. cerevisiae isolate EN14S01 ('standardized name': 'AMH') from (Peter et al., 2018). EN14S01 is a member of the highly diverged clade 17 thought to have branched early from all other $S$. cerevisiae isolates. Ancestral alleles defined by these two outgroups are in good agreement (Table 1). The population allele frequencies of the BY and RM variants across 1,011 S. cerevisiae species were obtained from (Peter et al., 2018). Predictions of variant effects were obtained using PROVEAN (Choi and Chan, 2015) (http://provean.jcvi.org) and are based on amino acid conservation and properties.

Variant changes resulting in scores of less than -2.5 are predicted to be deleterious. 


\section{Statistical analyses of allele effects}

532 All statistical analyses were conducted in R (https://www.r-project.org) version 4.0.4. The effect 533 of RM alleles on the expression of GFP-Gph1 (y) was estimated using the "lmer" function in the 534 lme4 package (Bates et al., 2015) for fitting mixed-effects linear models. In each case, one RM 535 allele (such as a block or a single variant, see below) was compared to strains with the BY allele 536 that were measured during the same runs in the plate reader. The models included the genotype 537 (BY vs. the given RM allele) as a fixed effect, plate and transformant as random effects (denoted 538 in parentheses), and the residual error $(\varepsilon)$ :

$539 y=($ plate $)+($ transformant $)+$ genotype $+\varepsilon$

540 The effects of genotype were estimated as slopes of the linear models. Significance was 541 calculated by using type I ANOVAs, as provided by the lmerTest package (Kuznetsova et al., 542 2017). These linear models and ANOVAs were performed to test the following effects:

543 1. The RM vs BY allele in both the BY or RM background

5442 . The various BY / RM combinations of the four blocks

5453 . The effect of each individual nonsynonymous variant compared to BY

\section{Testing for epistasis among the four "block" regions of $I R A 2$}

547 We tested for epistasis between blocks with a linear model. The model fitted the effects of all

548 four blocks simultaneously, along with all two-way, three-way, and the four-way interactions:

$549 y=($ plate $)+($ transformant $)+b 1: b 2+b 1: b 3+b 1: b 4+b 2: b 3+b 2: b 4+b 3: b 4+b 1: b 2: b 3+$ $550 b 1: b 2: b 4+b 1: b 3: b 4+b 2: b 3: b 4+b 1: b 2: b 2: b 4+\varepsilon$

551 We tested the significance of each term using a Type I ANOVA through sequential addition of 552 terms to the model above.

\section{Testing for epistasis between single nucleotide variant effects}

554 The single nucleotide variants were not engineered in all combinations, precluding the use of

555 interaction terms in a linear model. Instead, we asked whether the effects of single variants could 556 sum to the observed effect of their given multi-variant block or the full IRA2 RM allele. To

557 properly take into account measurement error across our experimental design, in which 558 individual transformants were run multiple times in several different runs of a plate reader, we 559 used bootstrapping. For each set of variants, we performed stratified sampling with replacement 560 across measurement plates, ensuring that each genotype was represented at the same sample size 
561 as in the full data. For each bootstrapped experiment, we fit the same models as above and

562 extracted the effect estimate. Single-variant effect estimates were summed to calculate the effect

563 they would be expected to have together under an additive model. We performed 10,000 of these

564 bootstraps to generate two distributions. The first distribution represented the difference between

565 BY and the given multi-variant RM allele, and the second distribution represented the sum of the

566 respective single-variant effects. Significance testing was performed by calculating the overlap

567 between the two distributions. If the central 95\% quantile range of the two distributions did not

568 overlap, we considered it significantly unlikely that the additive effects of the single variants are

569 able to account for the observed effect of the multi-variant allele. Thus, the less the two

570 bootstrapped distributions overlap, the stronger the evidence for epistatic interactions among the

571 given set of single variants.

\section{Code availability}

573 All analysis code is available at:

574 https://github.com/Krivand/Multiple-causal-DNA-variants-in-a-single-gene-affect-gene-expressi

575 on-in-trans

\section{Strain availability}

577 All strains constructed in this work are available on request.

\section{Supplementary Materials}

\section{Figures}

580 Figure S1: Gph1-GFP expression in chimeric IRA2 block alleles

581 Figure S2: Gph1-GFP expression in single-variant alleles

582 Figure S3: Gph1-GFP expression in block 1 alleles

\section{Tables}

584 Table S1: Strains

585 Table S2: Primers/Oligonucleotides

586 Table S3: Allele effects and linear models of full RM in BY or RM background

587 Table S4: Allele effects and linear models of all block combinations compared to BY

588 Table S5: Allele effects and linear models of single RM variants

589 Supplementary Table 6: Allele effects and linear models of block 1 RM, sub-block 1 RM, and

590 their single variants 


\section{Acknowledgements}

592 We thank Laura Sherer, Kaushik Renganaath, Laura Johnson, and Christian Brion for technical 593 contributions and discussions.

\section{Funding}

595 This work was supported by NIH grant R35GM124676 to FWA.

\section{References}

Adzhubei I, Jordan DM, Sunyaev SR. 2013. Predicting functional effect of human missense mutations using PolyPhen-2. Current protocols in human genetics Chapter 7:Unit7.20.

Albert FW, Bloom JS, Siegel J, Day L, Kruglyak L. 2018. Genetics of trans-regulatory variation in gene expression. eLife 7:e35471.

Albert FW, Kruglyak L. 2015. The role of regulatory variation in complex traits and disease. Nature Reviews Genetics 16:197-212.

Albert FW, Treusch S, Shockley AH, Bloom JS, Kruglyak L. 2014. Genetics of single-cell protein abundance variation in large yeast populations. Nature 506:494-497.

Ballester R, Marchuk D, Boguski M, Saulino A, Letcher R, Wigler M, Collins F. 1990. The NF1 locus encodes a protein functionally related to mammalian GAP and yeast IRA proteins. Cell 63:851-859. doi:10.1016/0092-8674(90)90151-4

Bates D, Mächler M, Bolker B, Walker S. 2015. Fitting Linear Mixed-Effects Models Using lme4. Journal of Statistical Software 67:1-48. doi:10.18637/jss.v067.i01

Bergoug M, Doudeau M, Godin F, Mosrin C, Vallée B, Bénédetti H. 2020. Neurofibromin Structure, Functions and Regulation. Cells 9:2365. doi:10.3390/cells9112365

Bernstein MR, Zdraljevic S, Andersen EC, Rockman MV. 2019. Tightly linked antagonistic-effect loci underlie polygenic phenotypic variation in C. elegans. Evolution Letters 3:462-473. doi:10.1002/ev13.139

Bloom JS, Boocock J, Treusch S, Sadhu MJ, Day L, Oates-Barker H, Kruglyak L. 2019. Rare variants contribute disproportionately to quantitative trait variation in yeast. eLife 8:e49212. doi:10.7554/eLife.49212 
618 Bloom JS, Ehrenreich IM, Loo WT, Lite T-LV, Kruglyak L. 2013. Finding the sources of missing heritability in a yeast cross. Nature 494:234-237.

620 Boyle EA, Li YI, Pritchard JK. 2017. An Expanded View of Complex Traits: From Polygenic to Omnigenic. Cell 169:1177-1186.

Brem RB, Yvert G, Clinton R, Kruglyak L. 2002. Genetic Dissection of Transcriptional Regulation in Budding Yeast. Science 296:752-755.

626 Brion C, Ambroset C, Sanchez I, Legras J-L, Blondin B. 2013. Differential adaptation to multi-stressed conditions of wine fermentation revealed by variations in yeast regulatory networks. BMC Genomics 14:681. doi:10.1186/1471-2164-14-681

D’angelo I, Welti S, Bonneau F, Scheffzek K. 2006. A novel bipartite phospholipid-binding module in the neurofibromatosis type 1 protein. EMBO Rep 7:174-179.

650 Fay JC. 2013. The molecular basis of phenotypic variation in yeast. Current opinion in genetics 651 \& development 23:672-677. 
652 Fehrmann S, Bottin-Duplus H, Leonidou A, Mollereau E, Barthelaix A, Wei W, Steinmetz LM,

653

654

655 Yvert G. 2013. Natural sequence variants of yeast environmental sensors confer cell-to-cell expression variability. Molecular Systems Biology 9:695-695.

Fidalgo M, Barrales RR, Ibeas JI, Jimenez J. 2006. Adaptive evolution by mutations in the FLO11 gene. Proceedings of the National Academy of Sciences 103:11228-11233. doi:10.1073/pnas.0601713103

Flint J, Mackay TFC. 2009. Genetic architecture of quantitative traits in mice, flies, and humans. Genome Res 19:723-733. doi:10.1101/gr.086660.108

Fournier T, Abou Saada O, Hou J, Peter J, Caudal E, Schacherer J. 2019. Extensive impact of low-frequency variants on the phenotypic landscape at population-scale. eLife 8:e49258. doi:10.7554/eLife.49258

Gerke J, Lorenz K, Cohen B. 2009. Genetic Interactions Between Transcription Factors Cause Natural Variation in Yeast. Science 323:498-501.

Gietz RD, Schiestl RH. 2007. High-efficiency yeast transformation using the LiAc/SS carrier DNA/PEG method. Nature Protocols 2:31-34.

Gil R, Seeling JM. 1999. Characterization of Saccharomyces cerevisiae strains expressing ira1 mutant alleles modeled after disease-causing mutations in NF1. Molecular and Cellular Biochemistry 202:109-118. doi:10.1023/A:1007058427880

Görner W, Durchschlag E, Martinez-Pastor MT, Estruch F, Ammerer G, Hamilton B, Ruis H, Schüller C. 1998. Nuclear localization of the C2H2 zinc finger protein Msn2p is regulated by stress and protein kinase A activity. Genes \& Development 12:586-597. doi:10.1101/gad.12.4.586

Großbach J, Gillet L, Clément-Ziza M, Schmalohr CL, Schubert OT, Barnes CA, Bludau I, Aebersold R, Beyer A. 2019. Integration of transcriptome, proteome and phosphoproteome data elucidates the genetic control of molecular networks. bioRxiv. doi:10.1101/703140

Grundberg E, Small KS, Hedman ÅK, Nica AC, Buil A, Keildson S, Bell JT, Yang T-P, Meduri E, Barrett A, Nisbett J, Sekowska M, Wilk A, Shin S-Y, Glass D, Travers M, Min JL, Ring S, Ho K, Thorleifsson G, Kong A, Thorsteindottir U, Ainali C, Dimas AS, Hassanali N, Ingle C, Knowles D, Krestyaninova M, Lowe CE, Di Meglio P, Montgomery SB, Parts L, Potter S, Surdulescu G, Tsaprouni L, Tsoka S, Bataille V, Durbin R, Nestle FO, O'Rahilly S, Soranzo N, Lindgren CM, Zondervan KT, Ahmadi KR, Schadt EE, Stefansson K, Smith GD, McCarthy MI, Deloukas P, Dermitzakis ET, Spector TD, The Multiple Tissue Human Expression Resource (MuTHER) Consortium. 2012. Mapping cis- and trans-regulatory effects across multiple tissues in twins. Nat 
688 Hoffman CS, Winston F. 1987. A ten-minute DNA preparation from yeast efficiently releases autonomous plasmids for transformation of Escherichia coli. Gene 57:267-272. doi:10.1016/0378-1119(87)90131-4

Holt LJ, Tuch BB, Villén J, Johnson AD, Gygi SP, Morgan DO. 2009. Global analysis of Cdk1

Horton RM, Hunt HD, Ho SN, Pullen JK, Pease LR. 1989. Engineering hybrid genes without the use of restriction enzymes: gene splicing by overlap extension. Gene 77:61-68. doi:10.1016/0378-1119(89)90359-4

Huh W-K, Falvo JV, Gerke LC, Carroll AS, Howson RW, Weissman JS, O’Shea EK. 2003. Global analysis of protein localization in budding yeast. Nature 425:686-691. doi:10.1038/nature02026

702 Kircher M, Witten DM, Jain P, O’Roak BJ, Cooper GM, Shendure J. 2014. A general framework for estimating the relative pathogenicity of human genetic variants. Nature Genetics

705 Kroymann J, Mitchell-Olds T. 2005. Epistasis and balanced polymorphism influencing complex trait variation. Nature 435:95-98. doi:10.1038/nature03480

Kuznetsova A, Brockhoff PB, Christensen RHB. 2017. lmerTest Package: Tests in Linear Mixed Effects Models. J Stat Soft 82. doi:10.18637/jss.v082.i13

Lanz MC, Yugandhar K, Gupta S, Sanford EJ, Faça VM, Vega S, Joiner AMN, Fromme JC, Yu

712 Lewis JA, Gasch AP. 2012. Natural Variation in the Yeast Glucose-Signaling Network Reveals a New Role for the Mig3p Transcription Factor. G3 - Genes $\mid$ Genomes $\mid$ Genetics

715 Longtine MS, Iii AM, Demarini DJ, Shah NG, Wach A, Brachat A, Philippsen P, Pringle JR. 716 1998. Additional modules for versatile and economical PCR-based gene deletion and 717 modification in Saccharomyces cerevisiae. Yeast 14:953-961. 718 doi:10.1002/(SICI)1097-0061(199807)14:10<953::AID-YEA293>3.0.CO;2-U

719 Luo G, Kim J, Song K. 2014. The C-terminal domains of human neurofibromin and its budding 
722 Lutz S, Brion C, Kliebhan M, Albert FW. 2019. DNA variants affecting the expression of numerous genes in trans have diverse mechanisms of action and evolutionary histories. PLoS Genet 15:e1008375. doi:10.1371/journal.pgen.1008375

Mackay TFC, Stone EA, Ayroles JF. 2009. The genetics of quantitative traits: challenges and 2019. Nucleic Acids Research 47:W636-W641. doi:10.1093/nar/gkz268

Martínez-Pastor MT, Marchler G, Schüller C, Marchler-Bauer A, Ruis H, Estruch F. 1996. The Saccharomyces cerevisiae zinc finger proteins Msn2p and Msn4p are required for transcriptional induction through the stress response element (STRE). The EMBO Journal 15:2227-2235. doi:10.1002/j.1460-2075.1996.tb00576.x

Metzger BPH, Wittkopp PJ. 2019. Compensatory trans-regulatory alleles minimizing variation in

.
3:448-461. doi:10.1002/ev13.137 
754 She R, Jarosz DF. 2018. Mapping Causal Variants with Single-Nucleotide Resolution Reveals

755

756

757

758

759

760

761

762

763

764

765

766

767

768

769

770

771

772

773

774 Biochemical Drivers of Phenotypic Change. Cell 172:478-490.e15. doi:10.1016/j.cell.2017.12.015

Sievers F, Wilm A, Dineen D, Gibson TJ, Karplus K, Li W, Lopez R, McWilliam H, Remmert M, Söding J, Thompson JD, Higgins DG. 2011. Fast, scalable generation of high-quality protein multiple sequence alignments using Clustal Omega. Mol Syst Biol 7:539. doi:10.1038/msb.2011.75

Sim N-L, Kumar P, Hu J, Henikoff S, Schneider G, Ng PC. 2012. SIFT web server: predicting effects of amino acid substitutions on proteins. Nucleic Acids Research 40:W452-7.

Smith EN, Kruglyak L. 2008. Gene-Environment Interaction in Yeast Gene Expression. PLoS Biology 6:e83.

Steinmetz LM, Sinha H, Richards DR, Spiegelman JI, Oefner PJ, McCusker JH, Davis RW. 2002. Dissecting the architecture of a quantitative trait locus in yeast. Nature 416:326-330.

Stojiljkovic M, Foulquié-Moreno MR, Thevelein JM. 2020. Polygenic analysis of very high acetic acid tolerance in the yeast Saccharomyces cerevisiae reveals a complex genetic background and several new causative alleles. Biotechnol Biofuels 13:126. doi:10.1186/s13068-020-01761-5

Sudarsanam P, Cohen BA. 2014. Single Nucleotide Variants in Transcription Factors Associate More Tightly with Phenotype than with Gene Expression. PLoS Genetics 10:e1004325.

Sutter NB, Bustamante CD, Chase K, Gray MM, Zhao K, Zhu L, Padhukasahasram B, Karlins E, Davis S, Jones PG, Quignon P, Johnson GS, Parker HG, Fretwell N, Mosher DS, Lawler DF, Satyaraj E, Nordborg M, Lark KG, Wayne RK, Ostrander EA. 2007. A Single IGF1 Allele Is a Major Determinant of Small Size in Dogs. Science 316:112-115. doi:10.1126/science. 1137045

Swaney DL, Beltrao P, Starita L, Guo A, Rush J, Fields S, Krogan NJ, Villén J. 2013. Global analysis of phosphorylation and ubiquitylation cross-talk in protein degradation. Nat Methods 10:676-682. doi:10.1038/nmeth.2519

Thevelein JM, de Winde JH. 1999. Novel sensing mechanisms and targets for the cAMP-protein kinase A pathway in the yeast Saccharomyces cerevisiae. Mol Microbiol 33:904-918. doi:10.1046/j.1365-2958.1999.01538.x

Tuller T, Carmi A, Vestsigian K, Navon S, Dorfan Y, Zaborske J, Pan T, Dahan O, Furman I, Pilpel Y. 2010. An Evolutionarily Conserved Mechanism for Controlling the Efficiency of Protein Translation. Cell 141:344-354. doi:10.1016/j.cell.2010.03.031 
Van Laere A-S, Nguyen M, Braunschweig M, Nezer C, Collette C, Moreau L, Archibald AL,

789

790

791

792 Haley CS, Buys N, Tally M, Andersson G, Georges M, Andersson L. 2003. A regulatory mutation in IGF2 causes a major QTL effect on muscle growth in the pig. Nature 425:832-836. doi:10.1038/nature02064

Visscher PM, Wray NR, Zhang Q, Sklar P, McCarthy MI, Brown MA, Yang J. 2017. 10 Years of GWAS Discovery: Biology, Function, and Translation. American Journal of Human Genetics 101:5-22.

Wang X, Kruglyak L. 2014. Genetic Basis of Haloperidol Resistance in Saccharomyces cerevisiae Is Complex and Dose Dependent. PLoS Genet 10:e1004894. doi:10.1371/journal.pgen.1004894

Wang Z, Qi Q, Lin Y, Guo Y, Liu Y, Wang Q. 2019. QTL analysis reveals genomic variants linked to high-temperature fermentation performance in the industrial yeast. Biotechnol Biofuels 12:59. doi:10.1186/s13068-019-1398-7

Wohler Sunnarborg S, Miller SP, Unnikrishnan I, LaPorte DC. 2001. Expression of the yeast glycogen phosphorylase gene is regulated by stress-response elements and by the HOG MAP kinase pathway. Yeast 18:1505-1514. doi:10.1002/yea.752

Wright FA, Sullivan PF, Brooks AI, Zou F, Sun W, Xia K, Madar V, Jansen R, Chung W, Zhou Y-H, Abdellaoui A, Batista S, Butler C, Chen G, Chen T-H, D'Ambrosio D, Gallins P, Ha MJ, Hottenga JJ, Huang S, Kattenberg M, Kochar J, Middeldorp CM, Qu A, Shabalin A, Tischfield J, Todd L, Tzeng J-Y, van Grootheest G, Vink JM, Wang Q, Wang Wei, Wang Weibo, Willemsen G, Smit JH, de Geus EJ, Yin Z, Penninx BWJH, Boomsma DI. 2014. Heritability and genomics of gene expression in peripheral blood. Nature Genetics 46:430-437.

Yvert G, Brem RB, Whittle J, Akey JM, Foss E, Smith EN, Mackelprang R, Kruglyak L. 2003. Trans-acting regulatory variation in Saccharomyces cerevisiae and the role of transcription factors. Nature Genetics 35:57-64.

Zhu J, Zhang B, Smith EN, Drees B, Brem RB, Kruglyak L, Bumgarner RE, Schadt EE. 2008. Integrating large-scale functional genomic data to dissect the complexity of yeast regulatory networks. Nature Genetics 40:854-861. 


\section{Supplementary Figures}

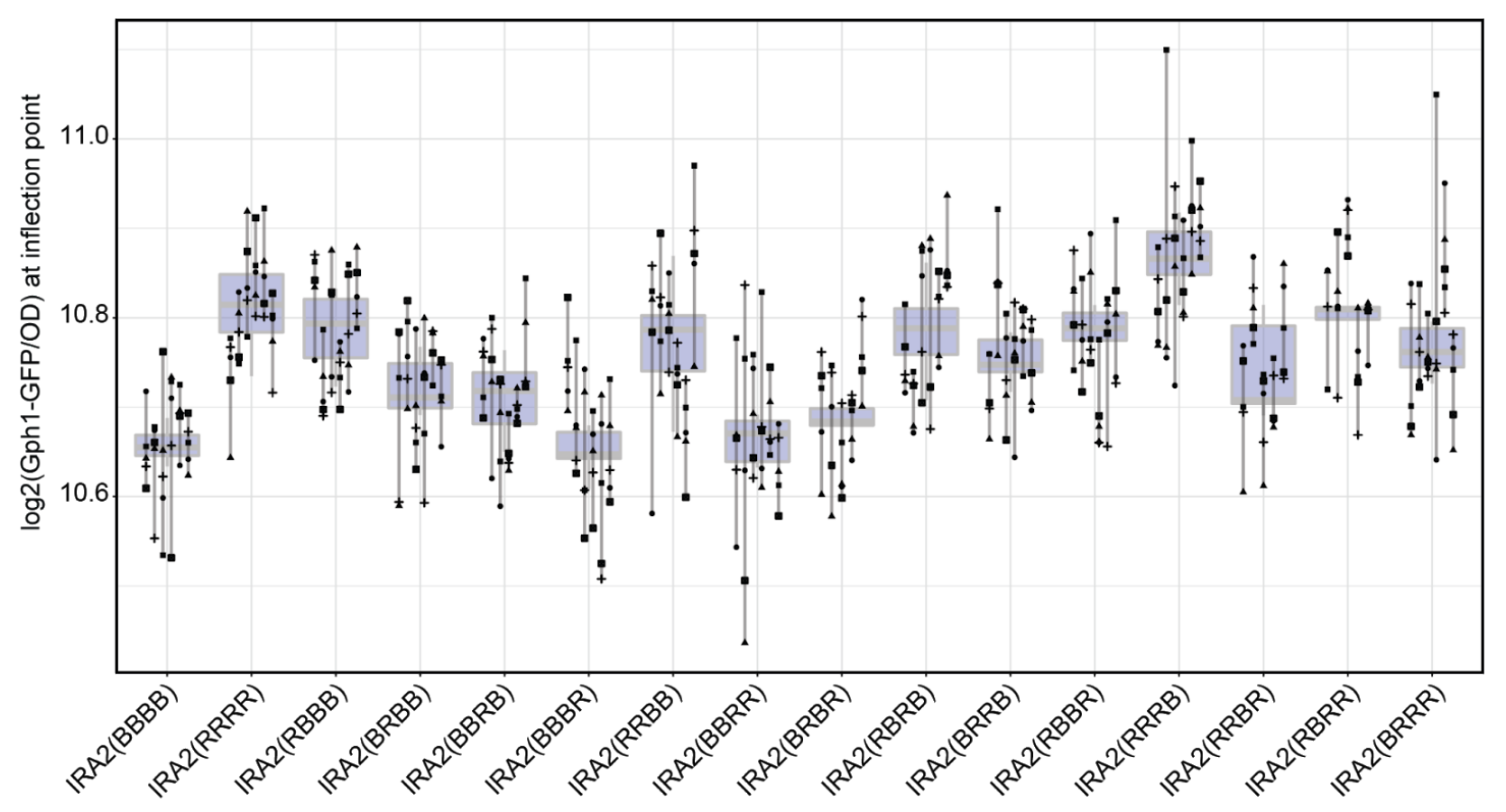

818 Figure S1 Measurement of Gph1-GFP expression in each of the 16 chimeric IRA2 block alleles.

819 Chimera alleles are indicated as four letters that are either B or R, indicating the BY or RM

820 allele, respectively, at blocks 1 through 4. Different shapes represent different plate reader runs.

821 Lines connect measurements of the same transformant. For each strain, five or six independent

822 transformants were phenotyped six times. The boxplots show the median as thicker central lines

823 and the first and third quartiles computed on averaged phenotypes per transformant. 


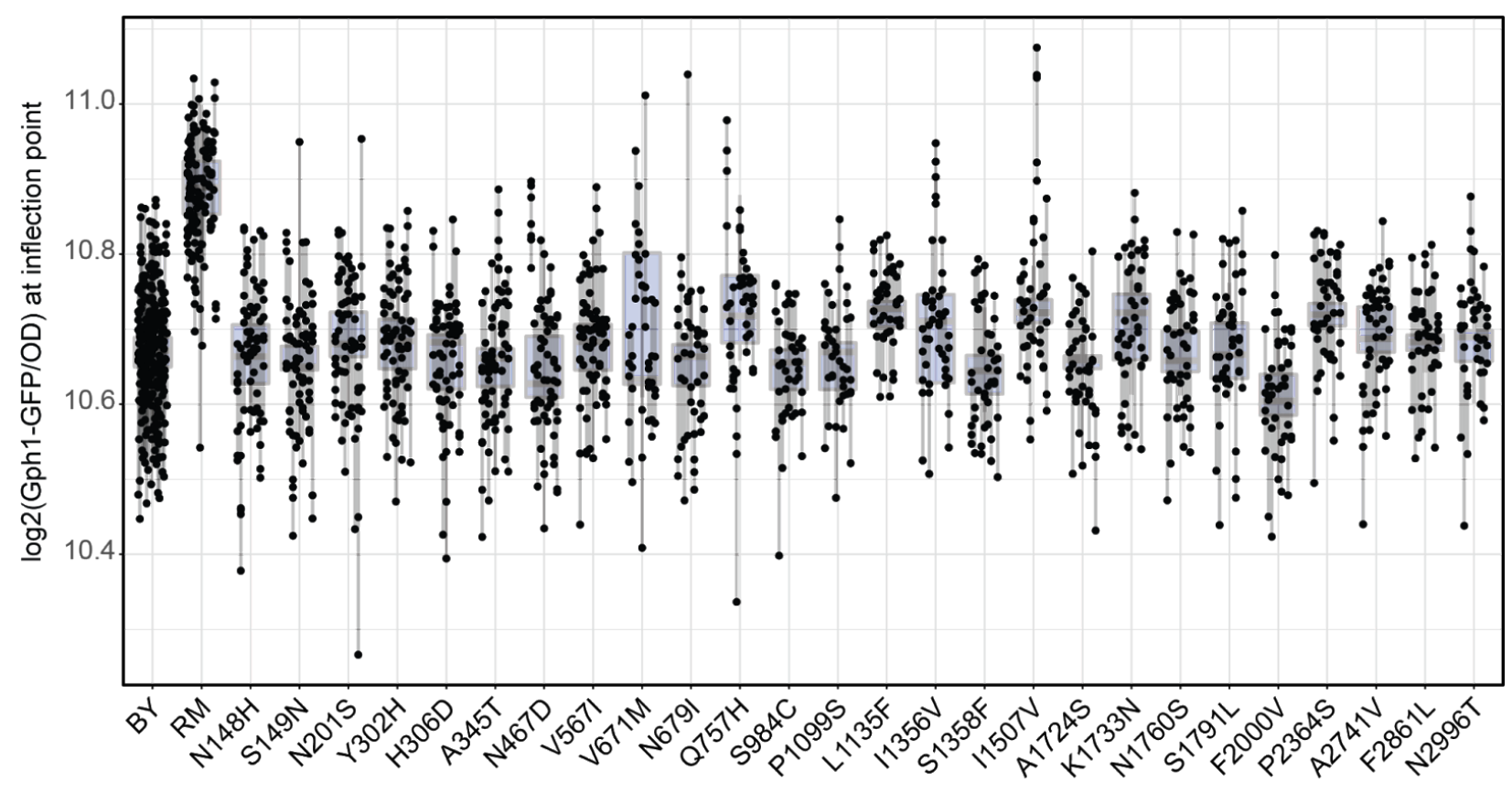

824 Figure S2 Measurement of Gph1-GFP expression in each single-variant strain. Lines connect 825 measurements of the same transformant. The IRA2(BY) allele is represented by 36 826 transformants, each measured 8 times. The IRA2(RM) allele is represented by 27 827 transformants, 9 of which were measured 8 times and 18 of which were measured 3 times. 828 Single-variant alleles $1-8$ are represented by 9 different transformants, each measured 8 times. 829 Single-variant alleles $9-26$ are represented by 9 transformants, each measured 5 times. The boxplots show the median as thicker central lines and the first and third quartiles computed on averaged phenotypes per transformant. 


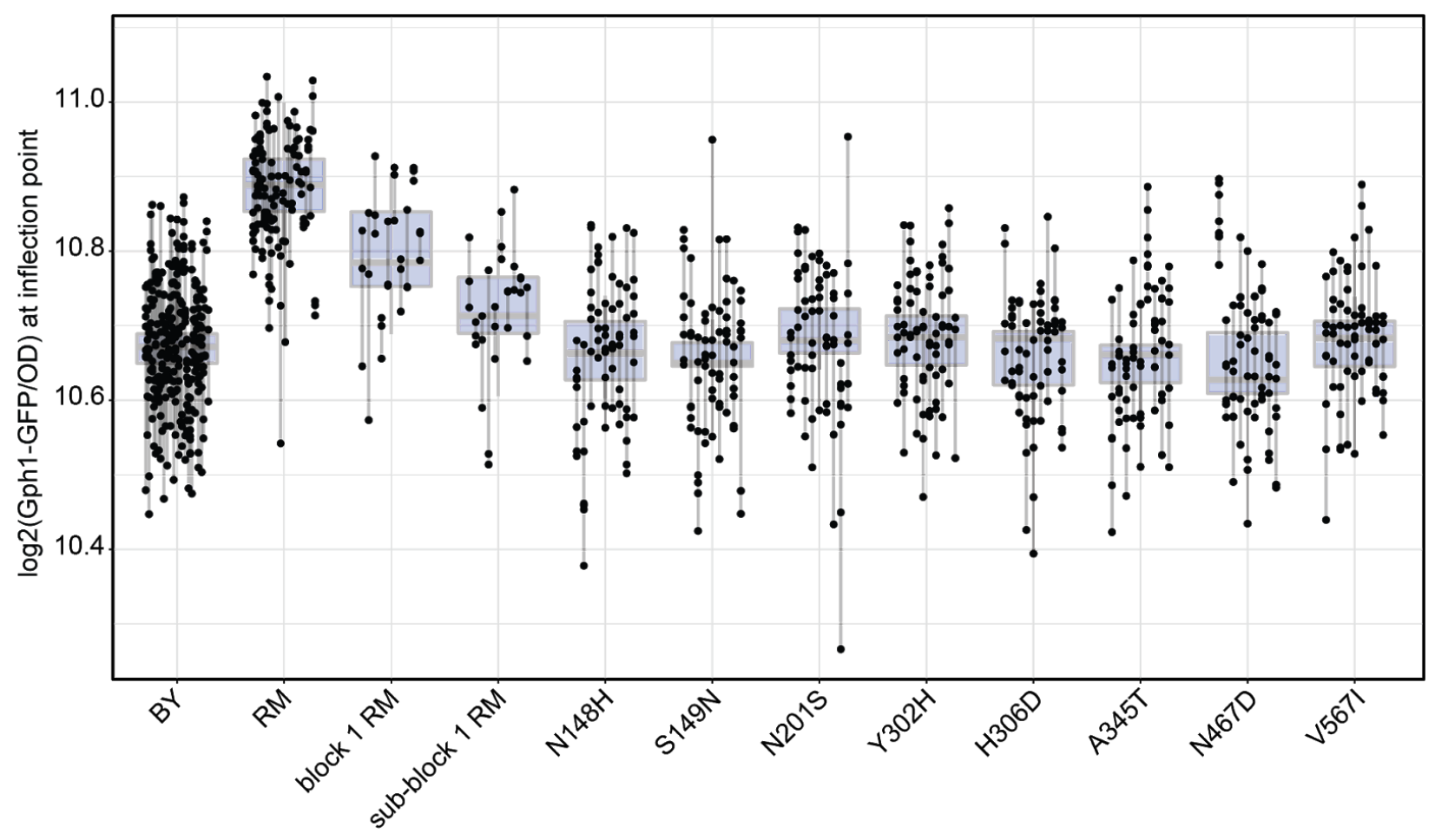

832 Figure S3 Measurement of Gph1-GFP expression of block1 variants, alone and in combination.

833 Lines connect measurements of the same transformant. The IRA2(BY) allele is represented by

83436 transformants, each measured 8 times. The IRA2(RM) allele is represented by 27

835 transformants, 9 of which were measured 8 times and 18 of which were measured 3 times.

836 Single-variant alleles $1-8$ are represented by 9 different transformants, each measured 8 times.

837 The block 1 and sub-block 1 alleles are each represented by 10 transformants measured 3

838 times. The boxplots show the median as thicker central lines and the first and third quartiles

839 computed on averaged phenotypes per transformant. 